\title{
One Plate-double Nutrient Endospore Activation Method
}

Bhagwan Narayan Rekadwad ${ }^{1,2,4, *}$, Juan M. Gonzalez ${ }^{3}$ and Chandrahasya N. Khobragade ${ }^{4}$

\author{
${ }^{1}$ Institute of Bioinformatics and Biotechnology, Savitribai Phule Pune University, Ganeshkhind, Pune \\ 411007, India; ${ }^{2}$ National Centre for Microbial Resource, National Centre for Cell Science, Pune 411007, \\ India; ${ }^{3}$ Institute of Natural Resources and Agrobiology, Spanish National Research Council, IRNAS- \\ CSIC, Avda. Reina Mercedes 10, 41012 Sevilla, Spain; ${ }^{4}$ School of Life Sciences, Swami Ramanand \\ Teerth Marathwada University, Nanded 431606, India \\ *For correspondence: rekadwad@gmail.com
}

[Abstract]This paper presents a simple, swift, improved and inexpensive one plate-double nutrient endospore activation method (ODNEAM) for activation of thermophilic endospore (thermospore). Revival and cultivation of dormant bacteria experiencing un-culturable state is a difficult task. Long-term preservation at low temperature $\left(4^{\circ} \mathrm{C}\right.$ or below) under laboratory conditions pushes most of the grampositive bacteria to form endospores and enter dormancy. After experienced dormancy and crossed specific time (more than a month) it's difficult for those thermospores to resurrect into a living cell. The effect of low temperature, a double quantity of nutrients and high humidity $(80 \%)$ was re-revaluated to foster germination of thermospores. Employed parameters successfully revived thermospores which experience dormancy or unable to initiate the germination process upon onset of favorable conditions. This method has crucial importance for activation of the dormant thermospore. Long-term preservation under low temperature or no sub-culturing at a fixed time interval of thermophilic bacteria may be a cause for least revival rate and the progressively diminished revival rate. The inactivation of cells after long-term preservation and probable loss to science would be avoided using this one plate-double nutrient endospore activation method. This is a tribute to Harold F. Foerster-A renowned microbiologist (1983).

Keywords: Endospore, Thermophiles, Extremophiles, Survival under low temperature, Hot springs

[Background] Thermophilic endospores (thermospores) are resistant structures. Thermospore represents resting phase/form of thermophilic bacteria which has the ability to survive under adverse conditions over a long time period and revive after arrival of favorable conditions. According to the thermophilic nature of these Bacilli, these spores are named "thermospores". Thermophilic bacteria Gram-positive and few Gram-negative would initiate process of formation of endospores upon onset of adverse conditions such as high or low temperature, salt, nutrients, water activity, oxic and anoxic conditions. Thermospore can survive in natural harsh environment (hot springs, hydrothermal areas, acid mines, alkaline lakes, salt lakes, ocean waters and sun-heated soils) and artificial extreme environments (xenobiotic environments, dumping sites, heaters, geysers, compost piles and dry areas). According to the growth temperature, microorganisms can be classified into psychrophiles, mesophiles, thermophiles, extreme thermophiles and hyperthermophiles. Temperature is a prime factor for the 
growth and development of thermophilic Gram-positive Bacilli and Gram-negative Sporomusa spp.

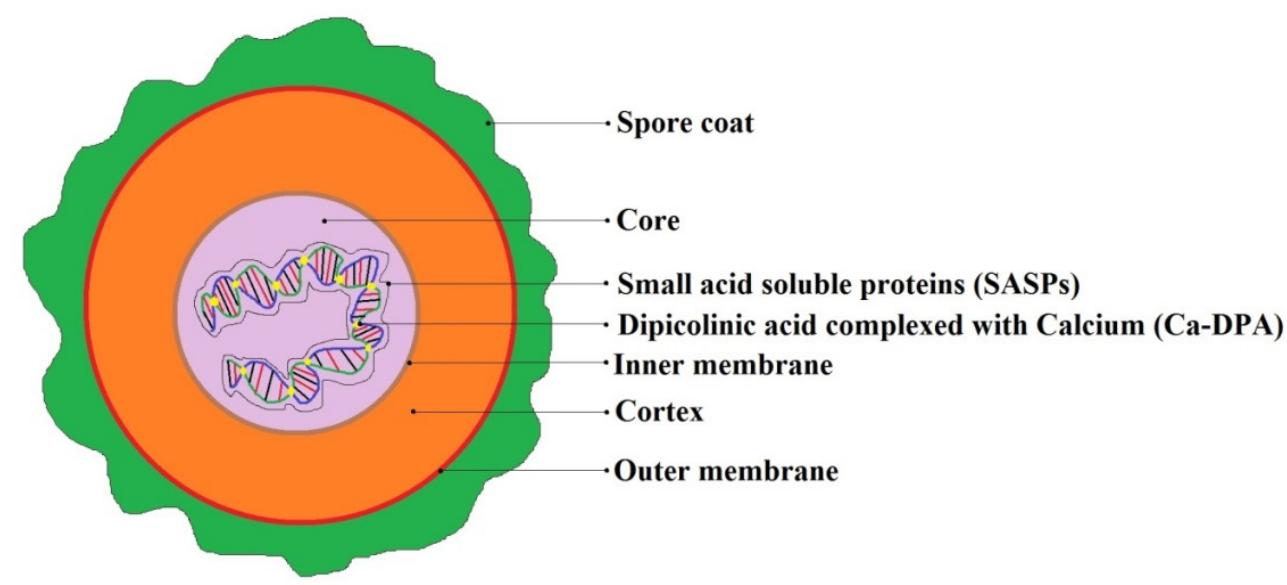

Figure 1. Typical endospore of bacteria

Experimental evidence shows that at non-optimal conditions thermophilic Bacilli have different adaption strategies. Whereas non-sporulating bacteria can produce solutes and heat shock protein for their survival. However, thermophilic bacteria and/or thermospore fail to revive after long-term preservation of without sub-culture longer than a month period. This gives a clue that revival of endospore requires optimum growth conditions which may differ than optimum growth parameter for exponentially growing cell. The revival of such dormant endospores at low temperature was primarily explored in the early 1980s by Harold F. Foerster (Foerster, 1983). He has proposed how to activate and re-grow endospore from Bacillus spp. In this protocol, we propose a modified procedure which consumes only one plate with double concentration of nutrients and applicable for thermophiles. This standardized protocol can be employed for the activation of mesophilic bacterial endospores. Moreover, we propose a specific incubation condition and simple protocol for activation of dormant. This is a tribute to Harold F. Foerster (1983).

\section{Materials and Reagents}

1. Polypropylene bottle (Tapper ware)

2. Glass slides

3. Eppendorf tube $(1.5 \mathrm{ml}$, Abdos Labtech Pvt. Ltd.)

4. Tryptone yeast extract agar (Himedia Pvt. Ltd., catalog number: M1272)

5. Nutrient agar (Himedia Pvt. Ltd., catalog number: M001)

6. Sporulation agar (Himedia Pvt. Ltd, catalog number: M1018.)

7. Physiological isotonic saline $(0.8 \%[\mathrm{w} / \mathrm{v}] \mathrm{NaCl})$

8. Endospore specific malachite green ( $1 \% \mathrm{w} / \mathrm{v})$ (Himedia Pvt. Ltd., catalog number: S020)

9. Safranin, $0.5 \%$ (w/v) (Himedia Pvt. Ltd., catalog number: S027)

Note: You could use the kit (below) as an alternative, it contains the same materials as 8 and 9. 
10. Schaeffer \& Fulton's Spore Stain-Kit (Himedia Pvt. Ltd., catalog number: K006-1KT) S028 Schaeffer \& Fulton's Spore Stain A S029 Schaeffer \& Fulton's Spore Stain B

\section{Equipment}

1. Laminar (Micro Filt India, model: MFI V 4x2)

2. Autoclave (Kumar Enterprises)

3. Incubator (Kumar Enterprises)

4. Cooling centrifuge (Remi C24)

5. Refrigerator (Godrej)

6. Olympus biological microscope (Olympus, model: CX33)

\section{Procedure}

A. Isolation of thermophilic bacteria from Hot Spring water

1. Take $1 \mathrm{~L}$ wide (appro. wide-mouth 2 inches) polypropylene bottle and wrap in a paper. Autoclave the polypropylene bottles at $121^{\circ} \mathrm{C}$ and $15 \mathrm{lbs}$ for $20 \mathrm{~min}$. And then irradiate autoclaved bottle under ultra-violet light in a laminar for $8 \mathrm{~min}$. Store the autoclaved and UV-irradiated bottles in sterile polythene bags until use. Use the autoclaved bottles within 2-4 days.

2. Collect hot water samples in an autoclaved sterile polypropylene bottle using composite sampling method. Collect four aliquots of water from hot spring at $15 \mathrm{~min}$ interval and prepare one composite sample (Rekadwad and Pathak, 2016). Leave approximately $2 \mathrm{~cm}$ airspace over water in a bottle and close cap tightly.

3. Immediately after sampling, transport collected water samples into laboratory within eight hours. Isolate bacteria on tryptone yeast extract agar and nutrient agar plate using spread plate method. Add $100 \mu$ hot spring water samples on nutrient agar surface and spread using glass rod.

4. Incubate plates for 24 to $96 \mathrm{~h}$ at $65^{\circ} \mathrm{C}$. After incubation, pick single and isolated colony for obtaining bacteria in pure culture. Multiple sub-cultures may be required for getting a pure culture of bacterium. Preserve isolated bacteria in the laboratory for further experiments.

B. Sporulation of bacteria

1. Streak $18-24 \mathrm{~h}$ activated culture of thermophilic bacteria on sporulation agar medium.

2. Incubate Petri-plates for $72-96 \mathrm{~h}$ at $65{ }^{\circ} \mathrm{C}$. Subsequently, preserve these bacteria at a lower temperature $\left(4^{\circ} \mathrm{C}\right)$. Preservation at a lower temperature for longer period of time ensures complete sporulation of the bacteria. Check the formation of endospore after a specific period (i.e., every 15 days), up to 2 months (see Section C. Endospore staining and observation).

3. Observe the slide for presence of endospore using Olympus biological microscope (CX33). First focus using 10x magnification and 10x eyepiece to locate the desired area for observation and 
then adjust to using 40x magnification and 10x eyepiece. For better visuals, add one drop of Cedar wood oil and observe slide for endospores using oil immersion lens (100x). It's recommended to use immersion oil to get 1,000 fold magnified image and achieve the best clarity.

4. Preserve culture in a refrigerator and maintain cool condition $\left(4^{\circ} \mathrm{C}\right)$ for 7 months.

C. Endospore staining and observation

1. After long-term storage at $4{ }^{\circ} \mathrm{C}$, take out cultured from the refrigerator.

2. Aseptically, add $1 \mathrm{ml}$ physiological sterile saline solution $(0.8 \%)$ in each Petri-plate. Allow soaking for $10-15 \mathrm{~min}$. If required, add $0.5 \mathrm{ml}$ more saline to prepare spore suspension. Gently scratch and prepare spore suspension.

3. Take a small aliquot of spore about $100 \mu \mathrm{l}$ using sterile pipette. Wash thrice in $200 \mu \mathrm{l}$ ice cold sterile saline solution or phosphate buffered saline $(\mathrm{pH} \mathrm{7.0)}$ by centrifuging at $21,952 \times g$ for 30 min at $4{ }^{\circ} \mathrm{C}$. Decant supernatant and save the pellet.

4. Prepare smear of spore suspension on a clean glass slide in $1.5 \mathrm{~cm} \times 1.5 \mathrm{~cm}$ area.

5. Heat fix slide by passing through flame for 1 to $3 \mathrm{~s}$. Check the temperature of slide tapping on your hand skin. If it wasn't too hot, pass it again for 1 more second.

Note: While tapping take caution and don't burn your hand.

6. Add endospore specific malachite green (1\%) dye and perform staining for $5 \mathrm{~min}$ on a boiling water bath as depicted in Figure 2A. Steam heating using boiling water bath may evaporate water in stain. Alternatively, use a thermostatic water bath for controlled steam heating. If required, add a few drops of malachite green stain to avoid drying of stain due to moist heat. Moist heat helps to penetrate malachite green dye in the cell wall and endospore coat will be strained in green color.

7. Use water to clean excess of stain. Gently, wash slide in a tray and air dry at room temperature. Later on, add a few drops of counterstain safranin which stains the bacterial cells in red. This will help to distinguish endospore for rest of vegetative body of bacterium. Wash slide gently and air dry as depicted in Figures 2B and 2C. You may use Schaeffer \& Fulton's Spore StainKit for endospore staining instead of malachite green (Spore Stain $A$ ) and counter stain safranin (Spore Stain B).

8. Observe slides for presence of endospores using Olympus biological microscope (CX33) as mentioned in the former step number (Step B3). 

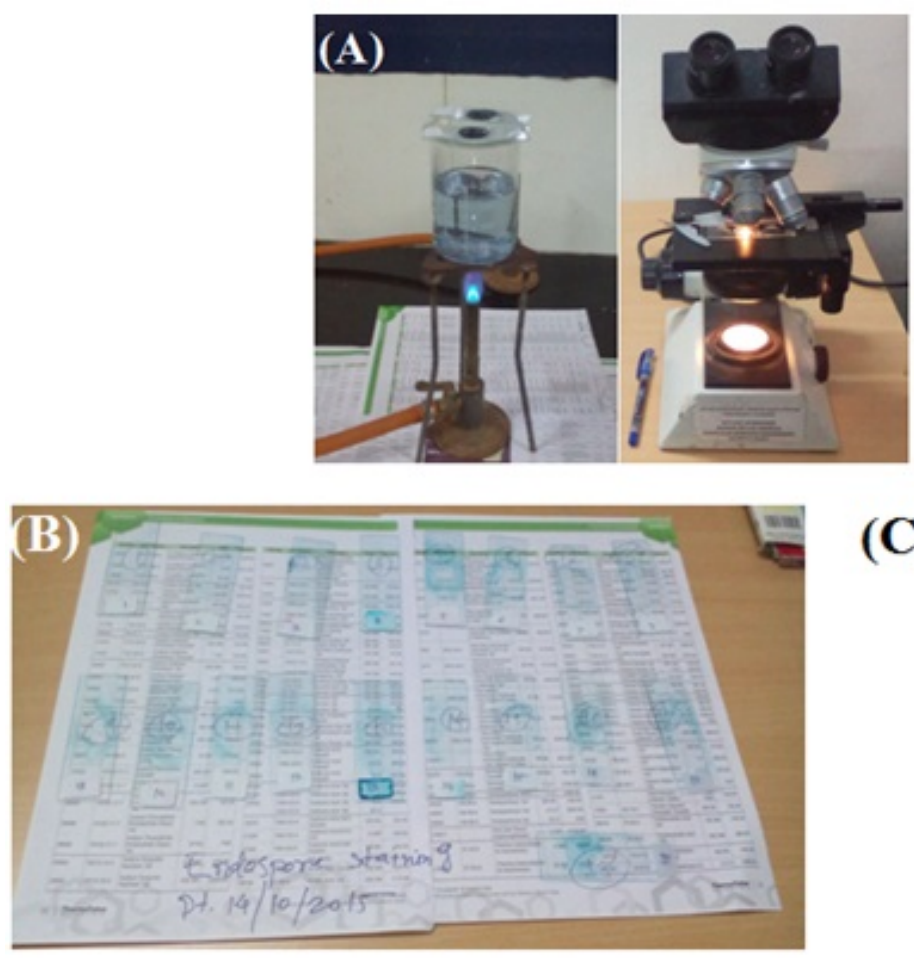

(C)

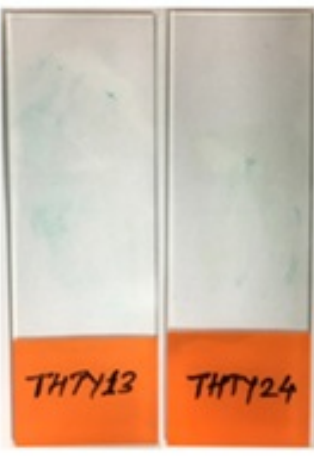

Figure 2. Endospore staining. A. Bacterial endospores staining-Slides were kept on the beaker. Heating to the water generates vapors which facilitate diffusion of warm malachite green in spore wall. B. Olympus Biological Microscope, CH20i-TR (Trinocular Version) was used for observation of endospores. C. Representative Slides were prepared for observation of endospore after staining under the microscope.

D. Direct sub-culturing stored bacterial culture (s)

Sub-culture bacteria as per standard operating procedure (SOP) in a microbiology lab. Aseptically take re-hydrated dried loop full bacterial culture as in the former steps (Steps C1 and C2) and streak on nutrient agar surface. Incubate Petri-plate containing sporulated bacterial culture at their optimum temperature for $72-96 \mathrm{~h}$. After completion of incubation period, check bacteria for viability or germination of endospores. 

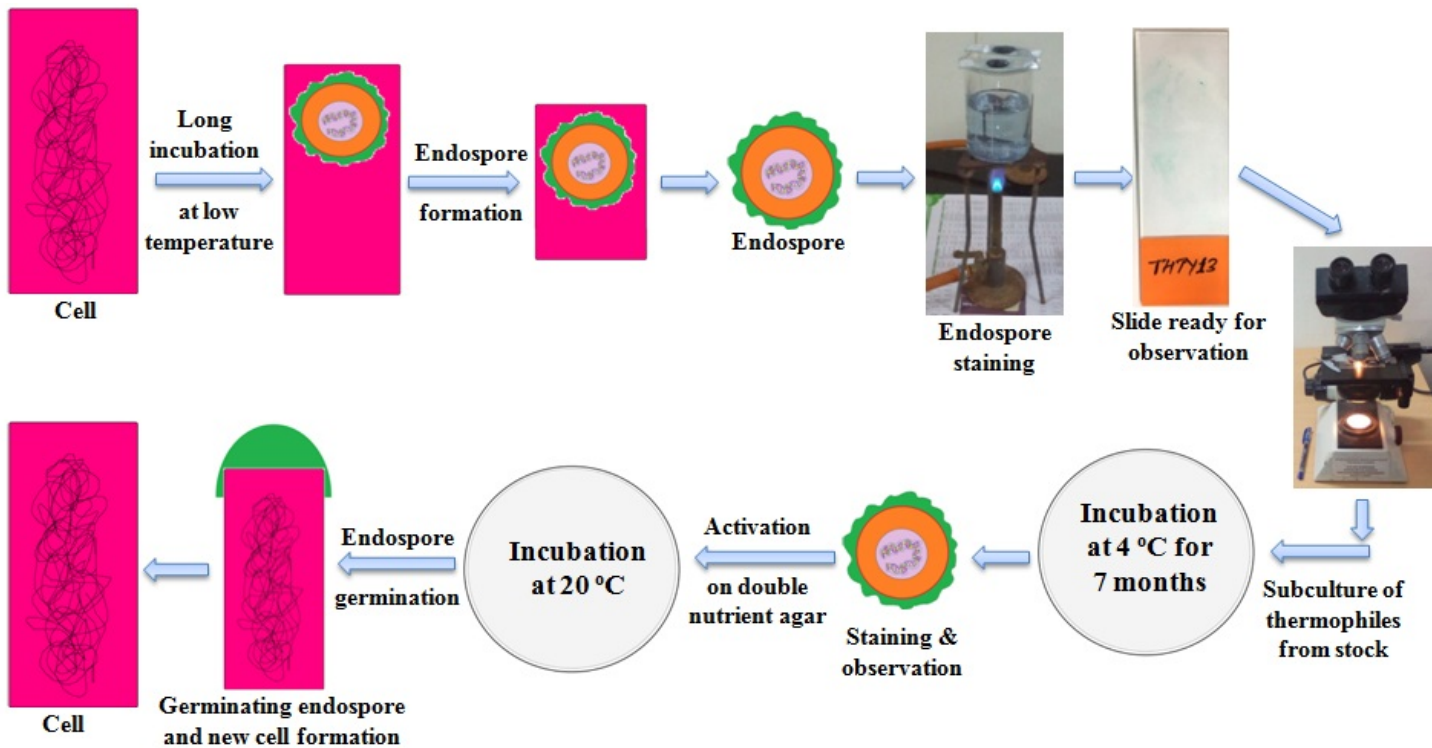

Activation

on double nutrient agar

Staining \&

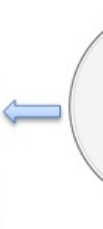

Incubation at $4{ }^{\circ} \mathrm{C}$ for

7 months from stock

Figure 3. Diagrammatic representation of sporulation and activation of thermophile endospore (thermospore) using a "one plate-double nutrient endospore activation method"

\section{E. Activation of endospores}

1. Use sporulated bacterial culture (Steps $\mathrm{C} 1$ and $\mathrm{C} 2$ ) for activation of thermospores. Scrap bacteria spores aseptically in laminar from preserved agar plates in sterile isotonic saline. Collect spore suspension in an autoclaved Eppendorf tube $(1.5 \mathrm{ml})$.

2. Centrifuge spore suspension at $4,000 \times \mathrm{g}$ for $2 \mathrm{~min}$ at room temperature to settle down agar debris. Use upper water layer as spore suspension.

3. Add $0.5 \mathrm{ml}$ spore suspension on nutrient agar surface. Spread spore suspension on double nutrient agar surface by gentle swirling in laminar platform. Double nutrient agar is $2 x$ standard concentration of nutrient agar. High volume ensures a more number of spores to be seeded and water quantity maintains high humidity inside Petri-plate.

4. Incubate plates in the refrigerator for $30 \mathrm{~min}$. This will help to lower media temperature quickly below $20^{\circ} \mathrm{C}$.

5. Later on, incubate plates at $20 \pm 2{ }^{\circ} \mathrm{C}$ in a cooling incubator until visible appearance of bacterial colonies on agar surface in a Petri plate.

F. The test for thermophily

"The test for thermophily" can be performed by incubating activated bacteria at $45^{\circ} \mathrm{C}$. Observe the growth of bacteria. Bacteria may show slower growth than its previously recorded incubation time (i.e., incubation time may have increased during activation process due to slower growth rate). Subculture bacteria and incubate at $65^{\circ} \mathrm{C}$ (i.e., at their original incubation temperature). Perform similar experiment in broth and record the results. Find out their optimum growth temperature as per SOP. 


\section{Data analysis}

As this protocol was designed as part of research. We have deduced our results like research paper. Readers or user are advised to use their own writing style for interpretation of results and as per requirements.

\section{Results}

A total of 89 isolated colonies of bacteria were randomly selected based on their optimum temperature in thermophilic range and distinguishable morphological features viz. color, size, configuration, margin, elevation and opacity were processed (Table 1). Pure cultures of thermophiles have been cultivated. These cultures were spread on sporulation agar medium which have forced bacteria to form thermospores. A graphical representation of the initiation of sporulation and activation processes of thermospores is depicted in Figure 3. Only 78 bacteria were grown and sporulated on sporulation agar medium after 72-96 $\mathrm{h}$ incubation time. Later on, bacteria have been transferred to refrigerator at $4{ }^{\circ} \mathrm{C}$ for 7 months. Most of the bacteria showed complete formation of endospores after 2 months of refrigeration. Endospore staining has confirmed the formation of endospore. Those cells showing clear formation of round endospores stained in green color were selected for further experiments. A total of 75 thermophilic bacteria showed complete formation of endospores at $4{ }^{\circ} \mathrm{C}$ after 7 months. Direct subculture confirmed non-viability of bacteria. Afterwards, the same dried bacterial mass was used for the endospore activation assay. Of 75 thermophiles, 58 isolates showed growth after activation using the above method. Furthermore, to confirm their thermophilic nature, "the test for thermophily was performed. It was recorded that bacteria show growth after 72-96 $\mathrm{h}$ incubation period at $45^{\circ} \mathrm{C}$. The incubation under low temperature apart from their optimum temperature and high humidity helped to activate endospores. The activated isolates were sub-cultured and incubated at $65^{\circ} \mathrm{C}$ to confirm their thermophilic character. Thus, 58 bacteria were successfully activated and retained their thermophilic character and passed the test for thermophily.

Table 1. Morphological features of representative thermophiles isolated from Unkeshwar hot spring (India)

\begin{tabular}{|c|c|c|c|c|c|c|c|}
\hline Strain & Color & $\begin{array}{l}\text { Size } \\
\text { (in } \mathrm{mm} \text { ) }\end{array}$ & Configuration & Margin & Elevation & $\begin{array}{l}\text { Gram } \\
\text { nature }\end{array}$ & Endospore \\
\hline THTY01 & Yellowish & 2 & Round & Entire & Raised & + & + \\
\hline THTY02 & White & 2 & Round & Entire & Flat & + & + \\
\hline THTY03 & Yellowish & 1 & Round & Smooth & Flat & + & + \\
\hline THTY04 & White & 2 & Irregular & Irregular & Flat & + & + \\
\hline THTY05 & White & 3 & Round with radiating & Smooth & Flat & + & + \\
\hline THTY06 & Yellowish & 1 & Concentric & Smooth & Umbonate & + & + \\
\hline THTY07 & Yellowish & 2 & Round & Irregular & Flat & + & + \\
\hline THTY08 & Light Pink & 1 & Concentric & Entire & Raised & + & + \\
\hline THTY09 & White & 1 & Irregular & Irregular & Convex & + & + \\
\hline
\end{tabular}


Table 1. Continued

\begin{tabular}{|c|c|c|c|c|c|c|c|}
\hline Strain & Color & $\begin{array}{l}\text { Size } \\
\text { (in mm) }\end{array}$ & Configuration & Margin & Elevation & $\begin{array}{l}\text { Gram } \\
\text { nature }\end{array}$ & Endospore \\
\hline THTY10 & White & 1 & Round & Wavy & Flat & + & + \\
\hline THTY11 & Yellowish & 2 & Round & Smooth & Umbonate & + & + \\
\hline THTY12 & White & 2 & Round & Irregular & Convex & + & + \\
\hline THTY13 & Yellowish & 1 & Round with radiating & Entire & Umbonate & + & + \\
\hline THTY14 & White & 1 & Concentric & Smooth & Flat & + & + \\
\hline THTY15 & White & 2 & Irregular & Irregular & Umbonate & + & + \\
\hline THTY16 & White & 3 & Concentric & Lobate & Flat & + & + \\
\hline THTY17 & White & 3 & Round & Smooth & Concave & + & + \\
\hline THTY18 & Reddish & 4 & Round with radiating & Smooth & Umbonate & + & + \\
\hline THTY19 & White & 4 & Concentric & Smooth & Concave & + & + \\
\hline THTY20 & White & 1 & Round & Smooth & Umbonate & + & + \\
\hline THTY21 & White & 2 & Roundwith Scalloped & Irregular & Flat & + & + \\
\hline THTY22 & White & 4 & Round & Wavy & Umbonate & + & + \\
\hline THTY23 & Light Pink & 2 & Concentric & Smooth & Flat & + & + \\
\hline THTY24 & White & 2 & Round with radiating & Smooth & Flat & + & + \\
\hline THTY25 & White & 2 & Round & Smooth & Crateriform & + & + \\
\hline THTY26 & White & 1 & Round with radiating & Smooth & Umbonate & + & + \\
\hline THTY27 & White & 1 & Concentric & Irregular & Flat & + & + \\
\hline THTY28 & White & 2 & Round with Scalloped & Irregular & Crateriform & + & + \\
\hline THTY29 & White & 1 & Round & Smooth & Convex & + & + \\
\hline THTY30 & White & 3 & Round with Scalloped & Smooth & Umbonate & + & + \\
\hline THTY31 & White & 1 & Round & Irregular & Crateriform & + & + \\
\hline THTY32 & White & 2 & Round & Smooth & Crateriform & + & + \\
\hline THTY33 & White & 1 & Round with Scalloped & Smooth & Flat & + & + \\
\hline THTY34 & White & 2 & Round with Scalloped & Irregular & Flat & + & + \\
\hline THTY35 & White & 2 & Round & Entire & Convex & + & + \\
\hline THTY36 & White & 1 & Round & Wavy & Crateriform & + & + \\
\hline
\end{tabular}

\section{Discussion}

Bacterial endospores were formed by certain groups of Gram-positive bacteria such as Bacillus, Clostridium and Gram-negative bacteria such as Sporomusa, etc. Endospore helps bacteria to survive under adverse environmental conditions. During the process of sporulation and activation experiments, bacteria undergo physiological and morphological transformations. The physiological barriers and molecular adaption provide resistance against multiple adverse factors, such as desiccation, heat, radiation, etc. (Laue et al., 2018). The massive changes in the environmental condition and scarcity in nutrient supply may damage/inactivate endospsores. This may lead to creation of non-functional resting structure or make cells as dead. It is believed that long-term preservation at low temperature without periodic passage (sub-culture) may help to maintain bacterial viability. However, experimental evidence shows that many bacteria are unable to survive after long-term storage or have poor doubling time or very slow growth rate. Additionally, long-term storage may lead to inactivation and loss of viability. The impacts of these conditions are poorly understood and require proved scientific evidence. Therefore, this protocol may act as alimelight for 
further research. The mechanism that triggers the germination process remains poorly understood. Moreover, focused research is required to understand the regulatory mechanisms and metabolic processes involved in spore formation and germination. It was hypothesized that environmental or artificial laboratory conditions may trigger the production of biomolecules such as small acid soluble proteins (SASPs), dipicolinic acid complex with Calcium (Ca-DPA) associated with genetic material in endospore core may initiate synergic metabolic pathways leading to the activation of endospores (de Vries, 2004). Therefore, it was assumed that combined action of humidity and nutrients may initiate of cascades of reactions leading to germination of thermospore (Brack, 1993; Kevnan, 1964; Gibbs, 1967).

Therefore in this resuscitation experiment, high humidity (more than $80 \%$ ) and high concentration of nutrient were maintained. Thermospores may have specific intracellular adaptations that maintain viscosity such a way that reduced water content, high nutrients and complex biomolecules generate structured molecular matrix. The solutes and biomolecules captured during formation of endospores may maintain molecular dynamism and intracellular viscosity which may assist endospore for survival under environmental stress (Cuecas et al., 2016). Additionally, using double nutrient concentration has facilitated growth. Ample supply of nutrients has avoided any potential limitation of essential nutrient sources. Hence, the revival of endospore requires optimum growth conditions which differed than optimum growth parameters of exponentially growing thermophile. Adopting this measure has better outcome such as best thermospore germination rate and revival of dormant thermophiles. This protocol might help to bring bacteria from dead stage. Moreover, this study provides a direction for further investigations about the mechanism of spore germination and resistance having relevance with preservation and activation of cultures.

\section{Conclusion}

The use of a simple and rapid procedure, i.e., one plate containing double nutrient, incubation at low temperature and presence of higher humidity (80\%) may trigger metabolic and regulatory pathways leading to the activation of endospore. Thus, more than $65 \%$ dormant thermophiles have been activated and revived in their original state successfully.

\section{$\underline{\text { Notes }}$}

1. There is a possibility to get false positive results. Keenly, observe endospores to differentiate from other structure in the cell.

2. Incubation at $65^{\circ} \mathrm{C}$ for 24 to $48 \mathrm{~h}$ ensures that bacterial colonies appeared are thermophiles.

3. Incubation time for bacteria used in this study was $72-96 \mathrm{~h}$. Incubation period may vary and depend on the type of bacterial phyla/species. In general, it equals to incubation period of first cycle of bacterium calculated before sporulation. It is suggested that author can observe and record results of every step either in scientific or vernacular language. 
4. Take caution while handing and preservation of cultures especially during preservation, aseptic handing, incubation or activation to avoid contamination by other microorganisms.

5. There will be accumulation of vaporized water or water drops during preservation of culture. This problem may arise due to preservation for long time and evaporation of water from media. Try to maintain cleanliness and hygiene in the refrigerator and preservation box. Periodically check preserved culture for accumulation of vaporized water and clean it.

\section{Acknowledgments}

BR thanks University Grants Commission, New Delhi (Government of India) for awarding postdoctoral fellowship and financial (Letter No. PDFSS-2013-14-ST-MAH-4350). The authors declare no conflict of interest. The authors acknowledge the Registrar, Swami Ramanand Teerth Marathwada University, Nanded, India (SRTMUN) and the Director, School of Life Sciences, SRTMUN, for providing the laboratory facilities.

\section{$\underline{\text { References }}$}

1. Brack, A. (1993). Liquid water and the origin of life. Orig Life EvolBiosph 23(1): 3-10.

2. Cuecas, A., Cruces, J., Galisteo-Lopez, J. F., Peng, X. and Gonzalez, J. M. (2016). Cellular Viscosity in Prokaryotes and Thermal Stability of Low Molecular Weight Biomolecules. Biophys $J$ 111(4): 875-882.

3. de Vries, Y. P. (2004). The role of calcium in bacterial spore germination. Microbes Environ 19(3): 199-202.

4. Foerster, H. F. (1983). Activation and germination characteristics observed in endospores of thermophilic strains of Bacillus. Arch Microbiol 134(3): 175-181.

5. Gibbs, P. A. (1967). The Activation of Spores of Clostridium bifermentans. Microbiology 46(2): 285-291.

6. Keynan, A., Evenchik, Z., Halvorson, H. O. and Hastings, J. W. (1964). Activation of bacterial endospores. J Bacteriol 88(2): 313-318.

7. Laue, M., Han, H. M., Dittmann, C. and Setlow, P. (2018). Intracellular membranes of bacterial endospores are reservoirs for spore core membrane expansion during spore germination. $\mathrm{Sci}$ $\operatorname{Rep~8(1):~} 11388$.

8. Rekadwad, B. and Pathak, A. (2016). First report on revelatory prokaryotic diversity of Unkeshwar hot spring (India) having biotechnological potential. Indian J Biotechnol 15:195-200. 